\title{
Sexualidade, reprodução e saúde: experiências de adolescentes que vivem em município do interior de pequeno porte
}

\author{
Sexuality, reproduction and health: experiences of adolescent \\ students living in a small city of the interior
}

Angélica Cristina Roza Pereira Vonk ${ }^{1}$

Claudia Bonan ${ }^{1}$

Kátia Silveira da Silva ${ }^{1}$

${ }^{1}$ Instituto Fernandes Figueira, Fiocruz. Av. Rui Barbosa 716, Flamengo. 22250-020 Rio de Janeiro RJ. angelicarozapereira@ hotmail.com

\begin{abstract}
This article aims to describe experiences of school adolescents of both sexes, living in a small city in the interior of the State of Rio de Janeiro, concerning affective-sexual life and sexual and reproductive health. A cross-sectional study was conducted with a structured questionnaire among 200 adolescents aged between 15 and 19 from public schools in Silva Jardim,. The girls' sexual initiation occurred between 15 and 19 and boys between 12 and 14. Boys started with partners from 12 to 19 years of age and girls with older partners. Girls received more information about sex than boys and talked more with partners about preventing pregnancy at the time of sexual initiation. Sources of information about sexuality and contraception are mainly from parents, with the pharmacy being the main location for the purchase of contraceptives. Information about sexually transmitted diseases is mainly received at school. The comparison of these results with those of other studies with adolescent students in large urban centers and populations that include youths outside school demonstrates similarities and dissimilarities between their experiences. Factors related to the socio-cultural and institutional context of small municipalities, gender and education help to understand them.
\end{abstract}

Key words Adolescents, Sex ality, Reproduction, Sexual and reproductive health
Resumo O objetivo deste artigo é escrever experiências de adolescentes escolares de ambos os sexos, de um município do interior do Rio de Janeiro, referentes à vida afetivo-sexual, à reprodução e à saúde sexual e reprodutiva. Estudo transversal com 200 adolescentes de escolas públicas de Silva Jardim, de 15 a 19 anos, com aplicação de questionário estruturado. A iniciação sexual das meninas ocorreu entre 15 e 19 anos e dos meninos entre 12 a 14 anos. Eles se iniciaram com parceiros de 12 a 19 anos e elas com parceiros mais velhos. Meninas receberam mais informações sobre relações sexuais do que meninos e conversaram mais com parceiros sobre prevenção de gravidez na ocasião da iniciação sexual. Fontes de informações sobre sexualidade e contracepção são principalmente os pais. A farmácia é o principal local de aquisição de contraceptivos. Informações sobre doenças sexualmente transmissiveis provêm principalmente da escola. A comparação desses resultados com os de outros estudos com adolescentes escolares de grandes centros urbanos e populações que incluem jovens fora da escola evidencia aproximações e distanciamentos entre suas experiências. Fatores relacionados ao contexto sociocultural e institucional de pequenos municípios, diferenciais de gênero e escolaridade ajudam a compreendê-las.

Palavras-chave Adolescentes, Sexualidade, Reprodução, Saúde sexual e reprodutiva 


\section{Introdução}

A promoção da saúde e os direitos sexuais e reprodutivos de adolescentes e jovens são temas de importante debate na sociedade brasileira, envolvendo autoridades do Estado, profissionais de saúde e educação, pesquisadores, organizações e movimentos sociais. Desde 1990, vem sendo construído um arcabouço jurídico-normativo que legitima esses direitos, tendo como marco o Estatuto da Criança e do Adolescente ${ }^{1}$, e, no âmbito da saúde, foram feitos investimentos em políticas, programas e ações para responder às necessidades de adolescentes e jovens ${ }^{2-4}$. Porém, indicadores de saúde mostram ainda lacunas entre a perspectiva de direitos e as condições de saúde, bem-estar e qualidade de vida desse grupo da população ${ }^{5-7}$.

A discussão sobre sexualidade, reprodução e saúde na adolescência tem sido objeto de extensa literatura científica na área da saúde coletiva, abrangendo questões como gravidez, aborto, contracepção, contracepção de emergência, doenças sexualmente transmissíveis e aids, violência, relação com os serviços de saúde, vivencias afetivo-sexuais, entre outras. Essa produção incluiu pesquisas multicêntricas que abrangem várias regiões e estados do país ${ }^{8,9}$ e um grande número de estudos locais (municípios, bairros, serviços). Estudos quantitativos analisam associações entre características sociodemográficas e práticas sexuais, praticas contraceptivas e abortivas $^{10-12}$ ou gravidez na adolescência ${ }^{13-15}$; analisam assistência pré-natal e resultados perinatais da gestação adolescente ${ }^{16,17}$, conhecimentos, informações e cuidados em saúde sexual e reprodutiva ${ }^{18-20}$ e relações dos adolescentes com serviços de saúde ${ }^{21,22}$. Estudos qualitativos exploram aspectos socioantropólogicos ${ }^{23,24}$, psicossociais e familiares $^{25-27}$, educativos ${ }^{28,29}$ e assistenciais, incluindo relações entre os adolescentes e profissionais de serviços de saúde ${ }^{30-33}$.

Essa produção científica tem destacado a heterogeneidade das vivências adolescentes, referentes à vida afetivo-sexual, à reprodução e aos cuidados com a saúde. Vários trabalhos mostram como diferenciais de gênero, classe social e raça/ cor influenciam as trajetórias dos adolescentes e jovens ${ }^{8,14,34}$ e os expõem de modo diferenciado a situações de vulnerabilidade social e de saúde $e^{30,35,36}$.

Quando se revisa a literatura brasileira sobre juventude, sexualidade e reprodução, um fato que chama a atenção é que a realidade de adolescentes que vivem em pequenas cidades do interior $\mathrm{e}$ de base rural é pouco estudada, e suas especifici- dades são pouco conhecidas. Diferenças socioculturais entre o modo de vida urbano e o rural podem implicar experiências e necessidades em saúde sexual e reprodutiva diferenciadas para os adolescentes. $\mathrm{O}$ trabalho apresentado neste artigo propõe-se a colocar no cenário do debate científico e da saúde publica a questão de como viver em municípios interioranos, de pequeno porte, com base socioeconômica predominantemente rural e com limitada infraestrutura em relação a serviços públicos pode condicionar as experiências juvenis.

O objetivo do estudo foi descrever experiências de adolescentes escolares de ambos os sexos de um município do interior do Estado do Rio de Janeiro, no que se refere à vida afetivo-sexual, à reprodução e aos cuidados com a saúde sexual e reprodutiva, incluindo iniciação afetiva e sexual, contracepção, gravidez, maternidade e paternidade, conhecimentos sobre doenças sexualmente transmissíveis e cuidados com a saúde sexual e reprodutiva.

\section{Sujeitos e métodos}

Trata-se de um estudo transversal realizado no município de Silva Jardim, no Estado do Rio de Janeiro. Silva Jardim foi escolhido por suas características de município de pequeno porte, com economia predominantemente rural, atendendo aos propósitos da pesquisa. O município está localizado a cerca de 110 quilômetros da capital do estado e, em 2009, sua população era de 22.224 habitantes. Em 2010, possuía 23 escolas públicas municipais (incluindo pré-escolas) e duas estaduais, não havendo estabelecimento de ensino privado na cidade. A rede pública de saúde é composta por um hospital, uma policlínica, um posto de saúde e oito unidades de saúde da família, todos sob gestão municipal. O setor privado é composto por três clinicas privadas.

Os sujeitos da pesquisa foram adolescentes matriculados em quatro escolas públicas, sendo duas estaduais e duas municipais. Foram incluídos indivíduos com idades de 15 a 19 anos, de ambos os sexos, independente de terem ou não iniciado vida sexual com parceiro. As escolas escolhidas foram aquelas que, segundo a Secretaria de Educação do Município, concentravam maior número de adolescentes nessa faixa etária, por terem ensino fundamental e médio, e educação para jovens e adultos.

Para a estimativa do tamanho amostral, considerou-se uma prevalência de $60 \%$ de atividade 
sexual entre os adolescentes de ambos os $\operatorname{sexos}^{37}$, com erro aceitável de 7\% e nível de 5\% de significância. Partiu-se de um universo de 1126 alunos de 15 a 19 anos matriculados nessas escolas, sendo obtido um tamanho amostral de 162 participantes. Para compensar possíveis perdas, o tamanho amostral foi acrescido de $20 \%$, ou seja, de, no mínimo, 192 adolescentes.

Os adolescentes foram convidados a participar da pesquisa e aqueles que se dispuseram a responder ao questionário foram incluídos, até completar o número de entrevistas necessárias.

A coleta de dados foi feita por meio de um questionário estruturado, pré-testado em estudo piloto, com 10 adolescentes de ambos os sexos. O instrumento continha questões que abrangiam informações sociodemográficas e familiares, e sobre a vida afetiva, o início da vida sexual, as práticas contraceptivas e os cuidados com a saúde sexual e reprodutiva. O trabalho de campo foi desenvolvido no período de junho a setembro de 2010, nos três turnos escolares.

As variáveis categóricas são apresentadas através de proporções. A análise estatística foi realizada através do teste de qui-quadrado $\left(\chi^{2}\right)$, sendo aplicada a correção de Yates, quando necessário. Utilizou-se o programa de Epi-info 3.58 para entrada e análise dos dados.

A pesquisa foi aprovada pelo Comitê de Ética em Pesquisa do Instituto Fernandes Figueira/ Fiocruz.

\section{Resultados}

Foram entrevistados 200 adolescentes escolares, sendo 127 do sexo feminino e 73 do sexo masculino. A média de idade foi de 16,6 anos para o total da amostra, sendo 16,3 para as mulheres e 16,5 para os homens. A escolarização das meninas foi significativamente maior que a dos meninos ( $p=0,008)$, embora não haja diferença significativa de idade entre os dois grupos (Tabela 1).

Nove meninas declararam-se unidas ou casadas, em contraste com os meninos, que eram todos solteiros. Todas elas viviam com parceiros em lar próprio. Em ambos os grupos, a maioria (mais de $80 \%$ ) vivia com um ou ambos os pais. Porém, um número maior de adolescentes do sexo masculino vivia sem os pais: a proporção de meninos nesta condição chegou a ser cinco vezes maior do que o de meninas. A maioria dos adolescentes do sexo masculino disse não ter religião, sendo observada diferença significativa entre os dois grupos $(p=0,027)$ (Tabela 1).
Mais do que as mulheres, os homens adolescentes possuíam atividades remuneradas $(\mathrm{p}=$ $0,0003)$. Esse fato pode ter influência no maior tempo de permanência na escola e na maior escolarização das mulheres (Tabela 1). À pergunta sobre que atividades exerciam, eles informaram serem auxiliares de mecânicos, pintores, ajudantes de mercado, babás e auxiliares de serviços gerais (dados não apresentados na tabela).

Uma grande proporção (62\%) de jovens não soube informar a renda familiar, o que comprometeu uma análise mais ampla das condições socioeconômicas. Entretanto, quase a metade das meninas e um terço dos meninos disseram receber bolsa-família (Tabela 1), o que sinaliza vulnerabilidade econômica. As informações sobre escolaridade e ocupação dos pais evidenciaram predomínio de baixa instrução e atividades com pouca qualificação, como embrulhador de doce, auxiliar de serviços gerais, faxineira, babá, do lar, pedreiro, mecânico, trabalhador rural e frentista (dados não apresentados na tabela). $\mathrm{Na}$ questão da cor, mais do que dois terços dos jovens se declararam pretos ou partos, sem diferença entre os grupos.

Em relação à vida afetiva e sexual, $85 \%$ dos adolescentes tiveram experiência de namorar ou "ficar", mas somente 26,8\% (IC95\% 19,5-35,5) das meninas e $38,4 \%$ (IC 95\% 27,4-50,5) dos meninos tinham experiência sexual. Os resultados seguintes referem-se exclusivamente a esse subgrupo de jovens iniciados sexualmente $(n=62)$.

Grande parte das meninas informou ter tido a primeira relação sexual entre os 15 e 19 anos, enquanto mais da metade dos meninos se iniciou mais jovem $(\mathrm{p}=0,014)$. Quase $80 \%$ dos homens tiveram a primeira experiência com adolescentes de 12 a 19 anos. A maioria das mulheres teve a primeira experiência sexual com pessoas acima dos 20 anos $(p<0,0001)$ e referiu que sua iniciação foi com o namorado $(p=0,006)$. Os meninos iniciaram-se sexualmente mais jovens e com pessoas com as quais "ficaram" (Tabela 2).

Perguntados se possuíam informações sobre relações sexuais antes da primeira experiência, mais da metade de todos os adolescentes respondeu que sim, porém as meninas tinham mais informações em comparação aos meninos ( $\mathrm{p}=$ $0,029)$. De modo mais frequente, também, elas haviam conversado com seus parceiros sobre gravidez antes da primeira relação sexual $(\mathrm{p}=0,015)$.

Sobre as fontes de informação na ocasião das primeiras relações sexuais, mães e/ou pais são os mais citados por adolescentes do sexo feminino e masculino. Amigos e namorados aparecem em 
Tabela 1. Dados sócio-demográficos e familiares de adolescentes escolares de Silva Jardim. 2010

\begin{tabular}{|c|c|c|c|c|}
\hline & $\begin{array}{c}\text { Total } \\
\mathbf{n}=200(\%)\end{array}$ & $\begin{array}{c}\text { Mulheres } \\
\mathrm{n}=127(\%)\end{array}$ & $\begin{array}{c}\text { Homens } \\
\mathbf{n}=73(\%)\end{array}$ & p valor \\
\hline \multicolumn{5}{|l|}{ Idade } \\
\hline $15-17$ & $149(74,5 \%)$ & $93(73,2 \%)$ & $56(76,8 \%)$ & \multirow[t]{2}{*}{$\mathrm{p}=0,586$} \\
\hline $18-19$ & $51(25,5 \%)$ & $34(26,8 \%)$ & $17(23,2 \%)$ & \\
\hline \multicolumn{5}{|l|}{ Cor } \\
\hline branca & $41(20,5 \%)$ & $27(21,3 \%)$ & $14(19,2 \%)$ & \multirow[t]{4}{*}{$\mathrm{p}=0,971$} \\
\hline preta (parda) & $137(68,5 \%)$ & $86(67,7 \%)$ & $51(69,8 \%)$ & \\
\hline outros (amarelo/indígena) & $10(5,0 \%)$ & $06(4,7 \%)$ & $04(5,5 \%)$ & \\
\hline não sabe & $12(6,0 \%)$ & $08(6,3 \%)$ & $04(5,5 \%)$ & \\
\hline \multicolumn{5}{|l|}{ Ano que está cursando } \\
\hline 1 a 5 série fundamental & $36(18 \%)$ & $18(14,2 \%)$ & $18(24,7 \%)$ & \multirow[t]{3}{*}{$\mathrm{p}=0,008$} \\
\hline 6 a 9 série fundamental & $45(22,5 \%)$ & $23(18,1 \%)$ & $22(30,1 \%)$ & \\
\hline 1 a 3 série médio & $119(59,5 \%)$ & $86(67,7 \%)$ & $33(45,2 \%)$ & \\
\hline \multicolumn{5}{|l|}{ Estado civil } \\
\hline solteira (o) & $191(95,5 \%)$ & $118(92,9 \%)$ & $73(100 \%)$ & \multirow[t]{2}{*}{$\mathrm{p}=0,048$} \\
\hline unida/casada (o) & $09(4,5 \%)$ & $09(7,1 \%)$ & $0 \%$ & \\
\hline \multicolumn{5}{|l|}{ Com quem mora } \\
\hline mãe/pai/ambos & $176(88 \%)$ & $114(89,8)$ & $62(84,9 \%)$ & \multirow[t]{3}{*}{$\mathrm{p}=0,0009$} \\
\hline outras pessoas (sem pai ou mãe) & $15(7,5 \%)$ & $04(3,1 \%)$ & $11(15,1 \%)$ & \\
\hline companheiro (a) & $09(4,5 \%)$ & $09(7,1 \%)$ & $0 \%$ & \\
\hline \multicolumn{5}{|l|}{ Tem atividade com renda atualmente } \\
\hline $\operatorname{sim}$ & $57(28,5 \%)$ & $25(19,7 \%)$ & $32(43,8 \%)$ & \multirow{2}{*}{$\mathrm{p}=0,0003$} \\
\hline não & $143(71,5 \%)$ & $102(80,3 \%)$ & $41(56,2 \%)$ & \\
\hline \multicolumn{5}{|l|}{ Renda Familiar } \\
\hline até 1 salário mínimo & $31(15,5 \%)$ & $24(18,9 \%)$ & $07(9,6 \%)$ & \multirow[t]{3}{*}{$\mathrm{p}=0,187$} \\
\hline mais de 1 até 3 salários mínimos & $41(20,5 \%)$ & $27(21,2 \%)$ & $14(19,2 \%)$ & \\
\hline não informou/não sabe & $125(62,5 \%)$ & $75(59,1 \%)$ & $50(68,5 \%)$ & \\
\hline \multicolumn{5}{|l|}{ Ajuda do governo ${ }^{* *}$} \\
\hline bolsa família & $84(42,0 \%)$ & $59(46,5 \%)$ & $25(34,3 \%)$ & \multirow[t]{3}{*}{$\mathrm{p}=0,313$} \\
\hline outros tipos de bolsas & $05(2,5 \%)$ & $03(2,4 \%)$ & $02(2,7 \%)$ & \\
\hline não recebe & $104(52 \%)$ & $62(48,8 \%)$ & $42(57,5 \%)$ & \\
\hline \multicolumn{5}{|l|}{ Religião*** } \\
\hline católica & $38(19 \%)$ & $31(24,4 \%)$ & $07(9,6 \%)$ & \multirow[t]{3}{*}{$\mathrm{p}=0,027$} \\
\hline protestante/evangélica & $65(32,5 \%)$ & $41(32,3 \%)$ & $24(32,8 \%)$ & \\
\hline nenhuma & $95(47,5 \%)$ & $54(42,5 \%)$ & $41(56,2 \%)$ & \\
\hline \multicolumn{5}{|l|}{ Área de moradia } \\
\hline urbano & $166(83 \%)$ & $100(78,7 \%)$ & $60(82,1 \%)$ & \multirow[t]{2}{*}{$\mathrm{p}=0,557$} \\
\hline rural & $34(17 \%)$ & $27(21,3 \%)$ & $13(17,9 \%)$ & \\
\hline
\end{tabular}

* 1 adolescente do sexo feminino e 2 do sexo masculino relataram renda familiar maior do que três salários mínimos. 3 adolescentes do sexo feminino e 4 do sexo masculino não informaram ou não sabem. ${ }^{* *+} 1$ adolescente do sexo feminino não informou e 1 do sexo masculino é praticante do candomblé.

segundo lugar para meninas e meninos. Chama a atenção a reduzida citação a professores e profissionais de saúde. As mídias também não aparecem com destaque. Não foram encontradas diferenças entre os grupos no que se refere a essa variável (Tabela 2).

Em relação à gravidez, apenas meninas declararam ter tido essa experiência. Nove adolescentes tinham histórico de gravidez: cinco tinham fi- lhos vivos, duas estavam grávidas no momento da entrevista e duas tinham histórico de abortamento espontâneo (Tabela 2). Foram encontradas diferenças estatísticas entre adolescentes do sexo feminino e masculino no que diz respeito a terem sido orientados sobre como evitar gravidez antes da primeira relação sexual: 100\% das meninas receberam informações, em contraste com $68 \%$ dos meninos $(\mathrm{p}=0,001)$ (Tabela 3$)$. As fon- 
Tabela 2. Dados sobre a vida afetiva e início da vida sexual de adolescentes escolares de Silva Jardim.2010

\begin{tabular}{|c|c|c|c|c|}
\hline & $\begin{array}{c}\text { Total } \\
\mathrm{n}=\mathbf{2 0 0}(\%)\end{array}$ & $\begin{array}{l}\text { Mulheres } \\
\mathrm{n}=127(\%)\end{array}$ & $\begin{array}{c}\text { Homens } \\
\mathrm{n}=73(\%)\end{array}$ & p valor \\
\hline \multicolumn{5}{|l|}{ Todos os adolescentes } \\
\hline \multicolumn{5}{|l|}{ Namorou/ficou } \\
\hline $\operatorname{sim}$ & $171(85,5 \%)$ & $109(85,8 \%)$ & $62(84,9 \%)$ & \multirow[t]{2}{*}{$\mathrm{p}=0,862$} \\
\hline não & $29(14,5 \%)$ & $18(14,2 \%)$ & $11(15,1 \%)$ & \\
\hline \multicolumn{5}{|l|}{ Transou } \\
\hline $\operatorname{sim}$ & $62(31 \%)$ & $34(26,8 \%)$ & $28(38,4 \%)$ & \multirow[t]{2}{*}{$\mathrm{p}=0,088$} \\
\hline não & $138(69 \%)$ & $93(73,2 \%)$ & $45(61,6 \%)$ & \\
\hline Adolescentes com experiência sexual & $n=62(\%)$ & $\mathrm{n}=\mathbf{3 4}(\%)$ & $\mathrm{n}=\mathbf{2 8}(\%)$ & \\
\hline \multicolumn{5}{|l|}{ Idade da primeira relação sexual } \\
\hline 12-14 anos & $25(40,3 \%)$ & $9(26,5 \%)$ & $16(57,1 \%)$ & \multirow[t]{2}{*}{$\mathrm{p}=0,014$} \\
\hline 15 a 19 anos & $37(59,7 \%)$ & $25(73,5 \%)$ & $12(42,9 \%)$ & \\
\hline \multicolumn{5}{|l|}{$\begin{array}{l}\text { Idade da pessoa com quem teve a primeira } \\
\text { relação sexual }{ }^{* *}\end{array}$} \\
\hline $12-19$ anos & $36(58,1 \%)$ & $14(41,2 \%)$ & $22(78,5 \%)$ & \multirow[t]{3}{*}{$\mathrm{p}<0,0001$} \\
\hline maior ou igual a 20 anos & $19(30,6 \%)$ & $18(52,9 \%)$ & $1(3,6 \%)$ & \\
\hline não informou/não sabia & $7(11,3 \%)$ & $2(5,9 \%)$ & $5(17,9 \%)$ & \\
\hline \multicolumn{5}{|c|}{ Pessoa com quem teve a primeira relação sexual ${ }^{* *}$} \\
\hline namorado/a & $37(59,7 \%)$ & $26(76,5 \%)$ & $11(39,3 \%)$ & \multirow[t]{3}{*}{$\mathrm{p}=0,006$} \\
\hline pessoa com quem ficou & $19(30,6 \%)$ & $6(17,6 \%)$ & $13(46,4 \%)$ & \\
\hline não especificou/não informou & $6(9,7 \%)$ & $2(5,9 \%)$ & $4(14,3 \%)$ & \\
\hline \multicolumn{5}{|l|}{ Tinha informação sobre relação sexual } \\
\hline $\operatorname{sim}$ & $38(61,3 \%)$ & $25(73,5 \%)$ & $13(46,4 \%)$ & \multirow[t]{2}{*}{$\mathrm{p}=0,029$} \\
\hline não & $24(38,7 \%)$ & $9(26,5 \%)$ & $15(53,6 \%)$ & \\
\hline \multicolumn{5}{|l|}{ Quem deu informação sobre relação sexual ${ }^{*}$} \\
\hline mãe/pai/ambos & $19(30,6 \%)$ & $13(38 \%)$ & $6(21,4 \%)$ & $\mathrm{p}=0,294$ \\
\hline amigos/colegas/parceiros/namorados & $11(17,7 \%)$ & $7(20,6 \%)$ & $4(14,3 \%)$ & $\mathrm{p}=0,832$ \\
\hline professores/profissionais de saúde & $3(4,8 \%)$ & $3(8,8 \%)$ & 0 & $\mathrm{p}=0,344$ \\
\hline tv/internet/outras mídias & $7(11,3 \%)$ & $3(8,8 \%)$ & $4(14,3 \%)$ & $\mathrm{p}=0,839$ \\
\hline \multicolumn{5}{|l|}{$\begin{array}{l}\text { Conversou sobre gravidez com o parceiro/a } \\
\text { antes da primeira relacão sexual }\end{array}$} \\
\hline $\operatorname{sim}$ & $41(66,1 \%)$ & $27(79,4 \%)$ & $14(50 \%)$ & \multirow[t]{2}{*}{$\mathrm{p}=0,015$} \\
\hline não & $21(33,9 \%)$ & $7(20,6 \%)$ & $14(50 \%)$ & \\
\hline \multicolumn{5}{|l|}{ Gravidez } \\
\hline Sim & $9(14,5 \%)$ & $9(26,5 \%)$ & 0 & \multirow[t]{2}{*}{$\mathrm{p}=0,010$} \\
\hline Não & $53(85,5 \%)$ & $25(73,5 \%)$ & $28(100 \%)$ & \\
\hline \multicolumn{5}{|l|}{ Filhos vivos } \\
\hline Sim & $5(8,1 \%)$ & $5(14,7 \%)$ & 0 & \multirow[t]{2}{*}{$\mathrm{p}=0,100$} \\
\hline Não & $57(91,9 \%)$ & $29(85,3 \%)$ & $28(100 \%)$ & \\
\hline
\end{tabular}

Os itens não informou/não sabia/não especificou não foram incluídos nas análises do p- valor.

tes dessas informações foram principalmente os pais, nos dois grupos. De fato, a prevenção da gravidez na primeira relação sexual foi alta: $82,3 \%$ declararam ter utilizado algum método contraceptivo nesse momento, sem diferenças estatísticas entre os sexos (Tabela 3). O método mais utilizado por ambos os sexos foi o preservativo masculino $(72,6 \% \%)$, seguido do coito interrompido $(8,1 \%)$ e a pílula anticoncepcional $(8,1 \%)$ (dados não apresentados em tabela).
A frequência de contracepção no momento da pesquisa superava os $90 \%$, sem diferença estatística entre os grupos (Tabela 3). O preservativo masculino era utilizado por $79 \%$ dos jovens, e as pílulas, por $24 \%$, sem menção a outros métodos (dados não apresentados em tabela).

Se pais e mães foram as principais fontes de informação sobre como evitar gravidez na ocasião da iniciação sexual, em momento posterior, entram em cena outras pessoas. Para as meni- 
nas, a orientação sobre o método utilizado no momento da pesquisa proveio principalmente de amigas, colegas e parceiros $(58,8 \%)$, seguidos dos pais $(38,2 \%)$ e professores, e profissionais de saúde $(35,3 \%)$. TV, internet e outras mídias apareceram em baixa proporção $(5,9 \%)$. No caso dos meninos, os pais continuam como as principais fontes de informação, mas há uma maior participação de amigos, colegas, professores e profissionais de saúde. Chama a atenção a diferença entre os sexos no que diz respeito ao acesso a informações pela TV, internet e revistas: $28,6 \%$ dos meninos citam esses meios e somente $5,9 \%$ das meninas $(\mathrm{p}=0,041)$ (Tabela 3).

A farmácia era o principal local de aquisição dos métodos utilizados pelos dois grupos, superando $75 \%$. Nenhum menino respondeu que obtinha método contraceptivo através de parceiros ou amigos, enquanto $32,3 \%$ das meninas o faziam ( $p$ $=0,012)$. Os serviços de saúde eram locais de ob-

Tabela 3. Práticas contraceptivas de adolescentes escolares de Silva Jardim. 2010

\begin{tabular}{|c|c|c|c|c|}
\hline Somente para quem teve relação sexual & $\begin{array}{c}\text { Total } \\
\mathrm{n}=62(\%)\end{array}$ & $\begin{array}{l}\text { Mulheres } \\
\mathrm{n}=34(\%)\end{array}$ & $\begin{array}{c}\text { Homens } \\
\mathrm{n}=28(\%)\end{array}$ & p valor \\
\hline \multicolumn{5}{|l|}{$\begin{array}{l}\text { Teve informação sobre evitar gravidez antes da } \\
\text { primeira relação sexual? }\end{array}$} \\
\hline $\operatorname{sim}$ & $53(85,5 \%)$ & $34(100 \%)$ & $19(67,9 \%)$ & $\mathrm{p}=0,001$ \\
\hline não & $9(14,5 \%)$ & 0 & $9(32,1 \%)$ & \\
\hline \multicolumn{5}{|l|}{ Quem te deu esta informação? ${ }^{*}$} \\
\hline mãe/pai/ambos & $35(56,4 \%)$ & $22(64,7 \%)$ & $13(46,4 \%)$ & $\mathrm{p}=0,443$ \\
\hline amigos/colegas/parceiros/namorados & $10(16,1 \%)$ & $7(20,6 \%)$ & $3(10,7 \%)$ & $\mathrm{p}=0,579$ \\
\hline professores/profissionais de saúde & $11(17,7 \%)$ & $7(20,6 \%)$ & $4(14,3 \%)$ & $\mathrm{p}=0,832$ \\
\hline tv/internet/outras mídias & $109(16,1 \%)$ & $4(11,8 \%)$ & $6(21,4 \%)$ & $\mathrm{p}=0,595$ \\
\hline \multicolumn{5}{|l|}{$\begin{array}{l}\text { Uso de alguma medida para se proteger de gravidez } \\
\text { na primeira relação sexual }\end{array}$} \\
\hline $\operatorname{sim}$ & $51(82,3 \%)$ & $30(88,2 \%)$ & $21(75 \%)$ & $\mathrm{p}=0,306$ \\
\hline não & $11(17,7 \%)$ & $4(11,8 \%)$ & $7(25 \%)$ & \\
\hline \multicolumn{5}{|l|}{ Utiliza método contraceptivo atualmente } \\
\hline $\operatorname{sim}$ & $57(91,9 \%)$ & $31(91,2 \%)$ & $26(92,9 \%)$ & $\mathrm{p}=0,821$ \\
\hline não & $5(8,1 \%)$ & $3(8,8 \%)$ & $2(7,1 \%)$ & \\
\hline \multicolumn{5}{|l|}{ Quem orientou sobre o método utilizado?* } \\
\hline mãe/pai/ambos & $24(38,7 \%)$ & $13(38,2 \%)$ & $11(39,3 \%)$ & $\mathrm{p}=0,955$ \\
\hline amigos/colegas/parceiros/namorados & $27(43,5 \%)$ & $20(58,8 \%)$ & $7(25 \%)$ & $\mathrm{p}=0,088$ \\
\hline professores/profissionais de saúde & $20(32,5 \%)$ & $12(35,3 \%)$ & $8(28,6 \%)$ & $\mathrm{p}=0,686$ \\
\hline tv/internet/outras mídias & $10(16,1 \%)$ & $2(5,9 \%)$ & $8(28,6 \%)$ & $\mathrm{p}=0,041$ \\
\hline \multicolumn{5}{|l|}{ Como adquire o método utilizado?* } \\
\hline servico de saúde/escola & $8(12,9 \%)$ & $1(2,9 \%)$ & $7(25 \%)$ & $\mathrm{p}=0,060$ \\
\hline parceiros/amigos & $11(17,7 \%)$ & $11(32,3 \%)$ & 0 & $\mathrm{p}=0,012$ \\
\hline farmácia & $47(75,8 \%)$ & $27(79,4 \%)$ & $20(71,4 \%)$ & $\mathrm{p}=0,786$ \\
\hline \multicolumn{5}{|l|}{$\begin{array}{l}\text { Conversa com parceiro/a sobre a necessidade de uso } \\
\text { de métodos para não engravidar }\end{array}$} \\
\hline sempre & $27(43,5 \%)$ & $21(61,7 \%)$ & $6(21,4 \%)$ & $\mathrm{p}=0,002$ \\
\hline algumas vezes & $16(25,8 \%)$ & $9(26,5 \%)$ & $7(25 \%)$ & \\
\hline nunca & $17(27,4 \%)$ & $4(11,8 \%)$ & $13(46,4 \%)$ & \\
\hline \multicolumn{5}{|l|}{ Uso de camisinha/preservativo nas relações sexuais ${ }^{* * *}$} \\
\hline nunca & $3(4,8 \%)$ & $2(5,9 \%)$ & $1(3,6 \%)$ & \\
\hline de vez em quando & $37(59,7 \%)$ & $22(64,7 \%)$ & $15(53,5 \%)$ & $\mathrm{p}=0,530$ \\
\hline sempre & $20(32,3 \%)$ & $9(26,5 \%)$ & $11(39,3 \%)$ & \\
\hline \multicolumn{5}{|l|}{$\begin{array}{l}\text { De quem é a responsabilidade de utilizar métodos } \\
\text { para não engravidar? }\end{array}$} \\
\hline principalmente de um dos parceiros & $7(11,3 \%)$ & $2(5,8 \%)$ & $5(8,9 \%)$ & $\mathrm{p}=0,280$ \\
\hline a responsabilidade é igualmente de ambos & $55(88,7 \%)$ & $32(94,2 \%)$ & $23(82,1 \%)$ & \\
\hline
\end{tabular}

* Para estas questões, há possibilidade de responder a mais de uma categoria e, por isso, o total é maior que $100 \%$. 2 adolescentes do sexo masculino não informaram. ${ }^{* * *} 2$ adolescentes, um de cada sexo, não informaram. 
tenção de métodos para $25 \%$ dos meninos, e apenas para 2,9\% das meninas, com uma significância limítrofe (Tabela 3). O uso da contracepção de emergência foi relatado por 10 adolescentes mulheres. O método é adquirido exclusivamente em farmácias e, geralmente, são os parceiros que vão comprar (dados não apresentados em tabela).

As adolescentes conversam com seus parceiros, mais frequentemente do que os meninos ( $\mathrm{p}=$ 0,002 ), sobre a necessidade de utilização de método para não engravidar. Quase metade dos meninos $(46,4 \%)$ disse que nunca conversava sobre o assunto com suas parceiras, enquanto somente $11,8 \%$ das meninas tinham essa atitude (Tabela 3 ).

A maioria dos adolescentes de ambos os sexos utilizava preservativos em suas relações sexuais de modo intermitente $(59,7 \%)$. Somente um terço dos meninos e um quarto das meninas afirmaram que sempre usavam a camisinha. ( $\mathrm{Ta}$ bela 3). À pergunta sobre de quem seria a responsabilidade de utilização dos métodos contraceptivos, $88,7 \%$ da amostra opinou que a responsabilidade é tanto do homem quanto da mulher, sem diferença entre os sexos.

Os cuidados dos adolescentes com relação à sua saúde sexual e reprodutiva são apresentados na Tabela 4. Os comportamentos de meninos e meninas mostraram-se muito semelhantes nos itens investigados. O HIV/Aids total é a doença sexualmente transmissível (DST) mais conheci- da $(88,5 \%)$, seguida de afecções como gonorreia, sífilis, cancro mole e outras. O conhecimento do preservativo como meio para evitar DST e HIV/ Aids é praticamente universal (95,5\%).

As principais formas de transmissão do HIV/ Aids são conhecidas por quase todos - relação sexual $(96 \%)$ e compartilhamento de seringas $(88,5 \%)$. Contudo, crenças sobre a transmissão através de carícias, beijos e compartilhamento de roupas e outros objetos ainda são muito difundidas. A categoria professores/profissionais de saúde apareceu como a principal fonte de informação dos adolescentes sobre as DST (65,5\%), mas, na verdade, os professores foram citados por $58,5 \%$ dos entrevistados, enquanto os profissionais de saúde, somente por 14\% (dados não apresentados em tabela). TV, internet e outras mídias também foram mais citadas quando se perguntou sobre informações em DST (Tabela 4) do que nas perguntas sobre informações acerca de sexualidade e contracepção (Tabela 3).

Aos adolescentes com experiência sexual foi perguntado se tinham algum histórico de DST, mas a resposta foi negativa em todos os casos.

\section{Discussão}

Dados obtidos nesta pesquisa referentes à renda familiar, recebimento de bolsas do governo, es-

Tabela 4. Conhecimentos em DST e HIV/AIDS de adolescentes escolares de Silva Jardim. 2010

\begin{tabular}{|c|c|c|c|c|}
\hline & $\begin{array}{c}\text { Total } \\
\mathbf{n}=\mathbf{2 0 0}(\%)\end{array}$ & $\begin{array}{c}\text { Mulheres } \\
\mathrm{n}=127(\%)\end{array}$ & $\begin{array}{c}\text { Homens } \\
\mathrm{n}=73(\%)\end{array}$ & p valor \\
\hline \multicolumn{5}{|l|}{ Doenças Sexualmente Transmissíveis que conhece ${ }^{*}$} \\
\hline HIV/AIDS & $177(88,5 \%)$ & $114(89,7 \%)$ & $63(86,3 \%)$ & $\mathrm{p}=0,855$ \\
\hline HPV & $10(5 \%)$ & $8(6,8 \%)$ & $2(2,7 \%)$ & $\mathrm{p}=0,469$ \\
\hline Gonorreia/sífilis/cancro mole/outras & $106(53 \%)$ & $78(61,4 \%)$ & $28(38,3 \%)$ & $\mathrm{p}=0,074$ \\
\hline \multicolumn{5}{|l|}{ Como você pode se prevenir das Doenças } \\
\hline \multicolumn{5}{|l|}{ Sexualmente Transmissíveis? } \\
\hline usando preservativo (camisinha) & $191(95,5 \%)$ & $122(96,1 \%)$ & $69(94,5 \%)$ & $\mathrm{p}=0,939$ \\
\hline não sabe/ não informou & $9(4,5 \%)$ & $5(3,9 \%)$ & $4(5,5 \%)$ & \\
\hline \multicolumn{5}{|l|}{ Como você pode pegar HIV/AIDS? } \\
\hline através de relação sexual & $192(96 \%)$ & $123(98,8 \%)$ & $69(94,5 \%)$ & $\mathrm{p}=0,908$ \\
\hline compartilhando seringa & $177(88,5 \%)$ & $118(92,9 \%)$ & $59(80,8 \%)$ & $\mathrm{p}=0,520$ \\
\hline através de carícias íntimas/beijando na boca/outras & $195(97,5 \%)$ & $123(96,8 \%)$ & $72(98,6 \%)$ & $\mathrm{p}=0,930$ \\
\hline \multicolumn{5}{|l|}{ Como você obteve informações sobre as Doenças } \\
\hline \multicolumn{5}{|l|}{ Sexualmente Transmissíveis?* } \\
\hline mãe/pai/ambos & $76(38 \%)$ & $50(39,3 \%)$ & $26(35,3 \%)$ & $\mathrm{p}=0,723$ \\
\hline professores /profissionais da saúde & $131(65,5 \%)$ & $88(69,2 \%)$ & $43(59 \%)$ & $\mathrm{p}=0,493$ \\
\hline amigos/colegas/companheiro & $46(23 \%) 88$ & $22(17,3 \%)$ & $24(33 \%)$ & $\mathrm{p}=0,050$ \\
\hline tv/internet/outras mídias & $(44 \%)$ & $64(50,3 \%)$ & $24(33 \%)$ & $\mathrm{p}=0,127$ \\
\hline
\end{tabular}

'Para estas questões, há possibilidade de responder a mais de uma categoria e, por isso, o total é maior que $100 \%$. 
colaridade e ocupação dos pais delineiam um perfil desses escolares como pertencentes a classes socioeconômicas mais baixas. Nessa amostra, $92,9 \%$ das mulheres eram solteiras, resultado distinto da pesquisa de representatividade nacional, a PNDS-2006, que encontrou $72,1 \%$ mulheres solteiras entre 15 e 19 anos. Mais de $80 \%$ nos dois grupos viviam com os pais, padrão que se repete em capitais brasileiras ${ }^{8,9}$.

$\mathrm{Na}$ amostra estudada, 31\% dos entrevistados haviam se iniciado sexualmente. Resultados semelhantes foram encontrados em outros estudos com adolescentes de escolas públicas de municípios da capital e do interior paulista e baiano ${ }^{12,20,38}$, porém na faixa etária de 11 a 19 anos. Quando são consideradas somente adolescentes do sexo feminino, o percentual de jovens com experiência sexual de nosso estudo $(26,8 \%)$ é inferior ao da PNDS-2006 (55,2\%). Isso corrobora um fato já demonstrado: que maior escolaridade está associada ao início mais tardio da atividade sexual e da vida conjugal ${ }^{8,39}$.

Diferenciais de gênero também influenciam a idade da iniciação sexual. Assim como este, outros estudos observaram que os jovens do sexo masculino vivenciam em idade mais precoce a sexualidade com parceiro ${ }^{9,12,30}$. A dupla moralidade sexual influencia outros aspectos da vivencia sexual dos adolescentes ${ }^{39}$. Em grande maioria, a iniciação sexual das meninas foi com namorados e homens mais velhos. Já quase a metade dos meninos iniciou-se em relações eventuais, com adolescentes. Escolares entrevistados em escolas de um município do interior de São Pau$l^{38}$ e jovens entrevistados em três grandes capitais brasileiras ${ }^{8}$ apresentaram comportamento semelhante. Isso nos leva a refletir que, para além das diferenças que possam existir nas experiências dos jovens - segundo vivam em grandes centros urbanos ou pequenos municípios do interior - a influência das normas de gênero nas trajetórias sexuais adolescentes é persistente. A reprodução de papéis e estereótipos de gênero, independentemente do lugar em que vivem e do grupo social a que pertencem, foi reportada em outros estudos ${ }^{8,9}$.

A utilização de medidas de prevenção de gravidez na primeira relação sexual entre os adolescentes estudados foi alta, com predominância do preservativo masculino. Este tem sido o método mais utilizado pelos jovens brasileiros no seu primeiro intercurso sexual ${ }^{12,19,20}$. No momento da pesquisa, o percentual de uso de contraceptivo mantinha-se elevado (91,9\%). No estudo de Alves e Lopes (2008), 82\% de adolescentes uni- versitários paulistas sexualmente ativos utilizavam método anticoncepcional. Os resultados encontrados em nossa pesquisa são elevados, se comparados à PNDS-2006, de representatividade nacional (66\%), e ao estudo de Martins et al. ${ }^{20}$, com escolares paulistas $(70 \%)$.

Os principais métodos utilizados pelos jovens de Silva Jardim seguem o padrão encontrado em outros estudos com adolescentes de capital e de interior ${ }^{12,38}$. O uso da camisinha foi referida por 93\% dos meninos e $67.6 \%$ das meninas estudados. Nenhum entrevistado soube informar se sua parceira utilizava pílula anticoncepcional. $\mathrm{Na}$ PNDS-2006, na faixa etária de 15 a 19 anos, foi encontrado um percentual inferior de uso de preservativo entre as mulheres: $17,5 \%$ entre as unidas e $32,6 \%$ entre as não unidas. Na pesquisa nacional, ao desagregar a informação por escolaridade, encontrou-se que a utilização de camisinha é diretamente proporcional aos anos de estudo, o que ajuda a compreender o alto percentual de respostas afirmativas no presente estudo.

Somente $26,5 \%$ de meninas e $39 \%$ de meninos do estudo disseram utilizar sempre a camisinha em suas relações sexuais. A maioria utilizava o método de modo esporádico $(59,7 \%)$. No estudo de Castro et al. ${ }^{9}, 58,3 \%$ dos adolescentes de escolas públicas da cidade do Rio de Janeiro, 66,3\% de Florianópolis, 61,1 \% de Goiânia e $59.9 \%$ de Cuiabá disseram utilizar sempre o preservativo, resultados diferentes dos nossos. Apesar do conhecimento generalizado da utilidade da camisinha na prevenção de DST e de gravidez, o uso consistente desse método entre os adolescentes de Silva Jardim é baixo e seria necessário investigar as barreiras que dificultam a adoção dessa prática. Nas 14 capitais estudadas por Castro et al. ${ }^{9}$, o principal motivo alegado pelos adolescentes para não utilizarem sempre o preservativo foi não terem "a camisinha à mão" sempre que precisam. Essas dificuldades podem ser acentuadas em cidades interioranas de pequeno porte. Barreiras geográficas, institucionais, econômicas ou culturais que limitam o acesso podem ser mais acentuadas fora dos grandes centros urbanos. Receio do acolhimento e situações de conflito nos serviços de saúde, além do medo de revelar suas práticas sexuais para profissionais de saúde, empregados de farmácia, família ou comunidade podem ser empecilhos às práticas preventivas em saúde sexual e reprodutiva ${ }^{23,30,32}$.

Assim como foi encontrado na PNDS-2006, o principal local de aquisição dos diversos métodos contraceptivos pelos adolescentes de ambos os sexos era a farmácia. A participação dos servi- 
ços de saúde foi ínfima, principalmente para as meninas $(2,9 \%)$. Na pesquisa nacional, cerca de um quarto das jovens recorria aos serviços de saúde para obter métodos. Condições mais difíceis de privacidade e anonimato em pequenas cidades podem ser obstáculos para as meninas, quando se trata de buscar assistência à saúde sexual e reprodutiva.

Foi significativa a diferença entre os sexos no que diz respeito a dialogar com o parceiro sobre gravidez, sendo essa uma prática mais comum entre as mulheres adolescentes. Segundo Heilborn et $a .^{8}$, a construção cultural da masculinidade contribui para uma atitude menos dialógica dos homens em relação a temas como contracepção e também há maior habilidade das mulheres para tal conversa, ligada à representação da mulher como provedora dos cuidados com os outros. Entretanto, buscar o diálogo não é sinônimo de facilidade na negociação da prevenção com o parceiro, já que as jovens referiram menor uso de camisinha. O uso menos consistente do preservativo masculino nas relações sexuais entre adolescentes do sexo feminino e o fato de as jovens terem pouco poder de negociação frente a seus parceiros poderiam contribuir para isso ${ }^{20}$.

Nove meninas entre aquelas que haviam se iniciado sexualmente $(26,5 \%)$ tiveram experiência de gravidez, e nenhum menino quis ou soube informar sobre gravidez de suas parceiras. Estudo com adolescentes de escolas públicas da Bahia, encontrou $18,1 \%$ de história de gravidez entre as meninas com vida sexual ${ }^{12}$. Um estudo de base populacional em município de porte médio do Rio Grande do $\operatorname{Sul}^{40}(32,6 \%)$ e outro analisando o banco de dados nacionais da PNDS-2006 ${ }^{41}$ encontraram percentuais mais elevados de história de gravidez entre as jovens sexualmente ativas (32,6\% e 35,6\%, respectivamente). A diferença entre os resultados dos estudos com escolares e aqueles de base populacional pode ser compreendida quando há evidencias de que a menor incidência de gravidez na adolescência tem sido relacionada a mais anos de estudos e à matrícula na escola $a^{8,14,34,42}$.

Chama à atenção a quase ausência de relatos sobre aborto neste estudo, sugerindo que essa informação tenha sido omitida, pois a realidade do aborto entre adolescentes tem sido reportada em vários estudos baseados em informes sobre informações hospitalares ${ }^{7,9,43}$. Alves e Lopes ${ }^{19}$, em estudo quantitativo com adolescentes universitários paulistas, também não encontraram relatos de aborto, mas acreditam que pode ter havido omissão, pelas dificuldades que os jovens têm de discutir essa temática.
No contexto da iniciação sexual, as meninas tinham significativamente mais informações sobre relações sexuais e formas de evitar a gravidez do que os meninos, achado similar ao encontrado por outro estudo com escolares ${ }^{12}$. Em ambos os grupos, predominam como fonte de informações mães, pais ou ambos, seguidos de parceiros e amigos. A participação dos pais na vida afetiva e sexual dos adolescentes é, sem dúvida, de grande importância para essa faixa etária. Outros estudos ${ }^{12,14}$ mostraram associações positivas entre informações e apoio provenientes dos pais e/ou mães e práticas contraceptivas no período que circunda a primeira relação sexual. Em estudo realizado em município do interior de São Paulo, adolescentes escolares consideraram os pais como principal fonte de informação ${ }^{38}$. Profissionais de saúde e professores tiveram uma participação pífia nesse momento da trajetória dos escolares de Silva Jardim.

Ao longo do tempo, além de pais e mães, as fontes de informação e orientação sobre contracepção vão sendo mais diversificadas, com aumento importante da participação de parceiros e amigos e menos expressivo dos serviços de saúde (35,3\% para meninas e $28,6 \%$ para meninos), conforme pode ser percebido quando se pergunta quem orientou sobre o método que utilizavam no momento da entrevista. O papel limitado dos profissionais de saúde em informar e orientar os jovens é preocupante, pois a associação entre acesso a serviço de saúde e práticas contraceptivas mais consistentes tem sido demonstrada ${ }^{12}$.

A predominância de pais e amigos como fontes de informação relativa à vida sexual e contracepção também foi reportada por Castro et al. ${ }^{9}$, mas estudo com alunos de ensino médio em Aracaju encontrou que TV, rádio, revistas e jornais eram as principais fontes de informação sobre métodos contraceptivos ${ }^{44}$. Na pesquisa em Silva Jardim, as mídias aparecem de maneira modesta como fonte de informação em comparação às demais, sendo os meninos os que mais utilizam esses meios. Questões econômicas e dificuldades de maior anonimato e privacidade podem dificultar o acesso de jovens, com menos recursos econômicos e moradores de pequenas cidades, à internet e a múltiplos canais de TV, assim como a compra de revistas e jornais especializados. Tais dificuldades devem ser mais acentuadas para as meninas, das quais se exige maior recato e discrição em relação às expressões da sexualidade.

Em relação a informações sobre DST e Aids, no estudo com escolares de Silva Jardim, os professores e a escola se destacam, sendo citados por 
$58,5 \%$ dos adolescentes, enquanto os profissionais e serviços de saúde somente por 14\% (dados não apresentados em tabela). As mídias são também mais utilizadas para se ter acesso a essas informações (44\%). Em outro estudo com escolares de cidade do interior, $71 \%$ disseram que os professores eram a principal fonte de informação sobre essas doenças, seguidos pela televisão (55\%): para os autores, isso demonstra "a importância da escola como cenário privilegiado de acolhimento contínuo de adolescentes e jovens" ${ }^{\text {"38. }}$.

$\mathrm{Na}$ amostra estudada, o conhecimento do HIV/Aids é generalizado e, em menor proporção, são conhecidas outras DST como gonorreia e sífilis. O conhecimento da camisinha como forma de prevenção é quase universal entre meninos e meninas - cerca de 95\%. Este resultado é similar ao de uma pesquisa de representatividade nacional para o grupo de jovens entre 15-24 anos ${ }^{45}$. Entretanto, também pode ser evidenciada a persistência generalizada de crenças errôneas sobre as formas de transmissão do HIV/Aids, (carícias íntimas, beijo na boca). Isso sinaliza a necessidade de maior investimento nas habilidades de professores, profissionais de saúde, familiares e mídias para transmitir informações corretas e desconstruir mitos e preconceitos, pois só assim os adolescentes estarão preparados para vivenciar suas experiências sexuais sem riscos para sua saúde e para a saúde de seus parceiros(as).

Neste estudo, de maneira geral, profissionais e serviços de saúde foram pouco citados como fontes de informação e orientação nos diversos momentos da trajetória sexual e contraceptiva. Taquette et al. ${ }^{32}$, ao estudarem um centro especializado em atender adolescentes, na cidade do Rio de Janeiro, identificaram que conflitos permeavam as relações entre os profissionais e os jovens, sendo os mais comuns aqueles relacionados ao sigilo e à confidencialidade. As autoras ressaltaram a importância de maior conhecimento, por parte dos profissionais, das leis e das diretrizes que regem o atendimento aos adolescentes, de maior interação com os órgãos competentes e de uma reflexão bioética continuada nos serviços, para que possam enfrentar situações conflituosas e garantir os direitos dos adolescentes. A carência desses conhecimentos, interações e reflexões pode ser mais acentuada em contexto de cidades do interior, em função da histórica desigualdade de distribuição de recursos sociais de diversos tipos, cuja concentração encontra-se nas grandes cidades, segundo regiões geopolíticas.

É preciso considerar algumas limitações do estudo, associadas ao fato de que muitos jovens não se sentem à vontade para expor as experiências relacionadas à sua vida afetiva e sexual, por culpa, vergonha ou medo. Esses sentimentos podem ser mais acentuados em cidades do interior, onde as redes de conhecimento são mais densas, embora tenham sido garantidos o sigilo das informações e a privacidade das entrevistas. Portanto, é possível que haja subestimativas de informações, como proporção de jovens com atividade sexual, história de gravidez e, principalmente, aborto, já que este tema é cercado de tabus e censuras em nossa sociedade. Também não pode ser descartado que essas informações tenham sido influenciadas por normas de gênero que contribuem para que as jovens do sexo feminino omitam sua experiência sexual.

\section{Considerações finais}

Ao compararmos os resultados deste estudo com escolares de Silva Jardim àqueles de outras pesquisas com escolares de grandes centros urbanos ou com populações que incluem jovens fora da escola, vemos aproximações e distanciamentos entre suas experiências que não podem ser explicados linearmente. Para uma compreensão abrangente das experiências desses adolescentes do interior, do que elas têm de singular e de seus aspectos mais generalizáveis, é necessário observar como se articulam fatores relacionados ao contexto sociocultural e institucional de pequenos municípios, ao diferencial da escolaridade e a questões de gênero. Por exemplo, assim como escolares residentes em áreas urbanas, os jovens deste estudo apresentaram menor percentual de experiência sexual com parceiros, quando comparados com estudos comunitários ou de representatividade nacional. Do mesmo modo, as diferenças entre meninos e meninas no que diz respeito às características de idade e do parceiro da primeira relação sexual, dificuldades de negociar o sexo seguro e outras são semelhantes àquelas verificadas em cidades grandes.

As lacunas deixadas pelos serviços de saúde em relação a seu papel como fonte de informação e orientação e meio para obter métodos contraceptivos, já evidenciadas em outros estudos, foram mais acentuadas no Município estudado, principalmente para as meninas. É provável que, em contextos como este, haja maiores dificuldades em relação a sigilo, privacidade e acolhimento, devido às menores oportunidades de os profissionais de saúde se capacitarem em relação à assistência aos adolescentes. Acrescente-se a isso 
o fato de que parte desses profissionais podem fazer parte do círculo de conhecimentos dos jovens ou ter parentesco com eles.

Esses resultados ratificam a necessidade de diálogo permanente e estratégias de ações integradas entre serviços de saúde e comunidade escolar, incluindo também professores, alunos, pais e mães e outros atores da comunidade, aspecto que poderia ser favorecido pela menor dimensão das cidades interioranas. Seria necessário colocar na roda de conversações os preceitos bioéticos, legais e programáticos referentes à assistência à saúde reprodutiva e sexual dos adolescentes, o que poderia ser favorecido com políticas e inicia- tivas de autoridades públicas e organizações da sociedade civil, no sentido de maior interiorização de recursos sociais diversos - de comunicação e informação, de promoção, de participação e debate, de aprimoramento profissional.

Dessa forma, junto aos demais atores sociais, os serviços de saúde poderiam desempenhar um papel significativo para a garantia dos direitos sexuais e reprodutivos dos adolescentes, ampliando seus conhecimentos, fortalecendo suas práticas de cuidados e promovendo uma reflexão sobre as repercussões da desigualdade de gênero na saúde, no bem-estar e na qualidade de vida dos jovens.

\section{Colaboradores}

ACRP Vonk, C Bonan e KS Silva participaram da concepção do projeto, do tratamento e analise dos dados e da redação do artigo. ACRP Vonk responsabilizou-se pela coleta e criação do banco de dados. 


\section{Referências}

1. Brasil. Lei no 8069 , de 13 de julho de 1990. Dispõe sobre o Estatuto da Criança e do Adolescente e dá outras providências. Diário Oficial da União 1990; 16 jul.

2. Brasil. Ministério da Saúde (MS). Coordenação de Saúde da Criança e do Adolescente. Programa de Saúde do Adolescente: bases programáticas. Brasília: MS; 1996.

3. Brasil. Ministério da Saúde (MS). Área de Saúde do Adolescente e do Jovem. Marco teórico e referencial: saúde sexual e saúde reprodutiva de adolescentes e jovens. Brasília: MS; 2006.

4. Brasil. Ministério da Saúde (MS). Departamento de Ações Programáticas Estratégicas. Diretrizes Nacionais para a Atenção Integral à Saúde de Adolescentes e de Jovens na Promoção, Proteção e Recuperação da Saúde. Brasília DF: MS; 2010.

5. Brasil. Ministério da Saúde (MS). Boletim Epidemiológico Aids/DST ano VII, no 01. Brasília: MS; 2010. [acessado 2011 jan 10]. Disponível em: http://www. aids.gov.br.

6. Brasil. Ministério da Saúde (MS). PNDS 2006 - Pesquisa Nacional de Demografia e Saúde da Criança e da Mulher. Brasília DF: MS; 2008.

7. Monteiro MFG, Adesse L. Estimativas de aborto in duzido no Brasil e Grandes Regiões (1992-2005). Área técnica de Saúde da Mulher. Brasília: MS; 2006.

8. Heilborn ML, Aquino EML, Bozon M, Knauth DR, organizadores. O aprendizado da sexualidade: reprodução e trajetórias sociais de jovens brasileiros. Rio de Janeiro: Editora Garamond, Editora Fiocruz; 2006.

9. Castro MG, Abramovay M, Silva LB. Juventudes e sexualidade. Brasília: Unesco;2004.

10. Rocha CLA, Horta B, Pinheiro RT, Cruzeiro ALS. Use of contraceptive methods by sexually active teenagers in Pelotas, Rio Grande do Sul State, Brazil. Cad Saude Publica 2007; 23(12):2862-2868.

11. Pirotta KCM, Schor N. Intenções reprodutivas e práticas de regulação da fecundidade entre universitários. Rev Saude Publica 2004; 38(4):495-502.

12. Almeida MCC, Aquino EML, Gaffikinb L, Magnanic RJ. Uso de contracepção por adolescentes de escolas públicas na Bahia. Rev Saude Publica 2003; 37(5):566-575.

13. Chalem E, Mitsuhiro SS, Ferri CP, Barros MCM, Guinsburg R, Laranjeira R. Gravidez na adolescência: perfil sociodemográfico e comportamental de uma população da periferia de São Paulo, Brasil. Cad Saude Publica 2007; 23(1):177-186.

14. Aquino EML, Heilborn ML, Knauth D, Bozon M, Almeida MC, Araújo J, Greice M. Adolescência e reprodução no Brasil: a heterogeneidade dos perfis sociais. Cad Saude Publica 2003; 19(Supl. 2):S377S388.

15. Simões VMF, Silva AMM, Bettiol H, Lamy-Filho F, Tonial SR, Mochel EG. Características da gravidez na adolescência em São Luís do Maranhão. Rev Saude Publica 2003; 37(5):559-565.
16. Carniel EF, Zanolli ML, Almeida CAA, Marcillo AM. Características de mães adolescentes e de seus recém-nascidos e fatores de risco para a gravidez na adolescência em Campinas,SP, Brasil. Rev Bras Saúde Matern Inf 2006; 6(pt.4):419-426.

17. Gama SGN, Szwarcwald CL, Leal MC. Experiência de gravidez na adolescência, fatores associados e resultados perinatais entre puérperas de baixa renda. Cad Saude Publica 2002; 18(1):153-161.

18. Sousa MCR, Gomes KRO. Conhecimento objetivo e percebido sobre contraceptivos hormonais orais entre adolescentes com antecedentes gestacionais Cad Saude Publica 2009; 25(3):645-654

19. Alves AS, Lopes MHBM. Conhecimento, atitude e prática do uso de pílula e preservativo entre adolescentes universitários Rev Bras Enferm 2008; 61(1): 11-17.

20. Martins LBM, Paiva LHSC, Osis MJD, Sousa MH, Neto AMP, Tadini V. Fatores associados ao uso de preservativo masculino e ao conhecimento sobre DST/AIDS em adolescentes de escolas públicas e privadas do Município de São Paulo, Brasil. Cad Saude Publica 2006; 22(2):315-323.

21. Claro LBL, March C, Mascarenhas MTM, Castro IAB, Rosa MLG. Adolescentes e suas relações com serviços de saúde: estudo transversal em escolares de Niterói, Rio de Janeiro, Brasil. Cad Saude Publica 2006; 22(8):1565-1574.

22. Carvalho IE, Mello MB, Morais SS, Silva JLP. Fatores associados ao acesso anterior à gestação a serviços de saúde por adolescentes gestantes. Rev Saude Publica 2008;42(5):886-894.

23. Brandão, ER. Desafios da contracepção juvenil: interseções entre gênero, sexualidade e saúde. Cien Saude Colet 2009; 14(4):1063-1071.

24. Heilborn ML, Salem T, Rohden F, Brandão E. Aproximações socioantropológicas sobre a gravidez na adolescência. Horizontes Antropológicos 2002; 8(17): 13-45.

25. Silva L, Tonete VLP. A gravidez na adolescência sob a perspectiva dos familiares: Compartilhando projetos de vida e cuidado. Rev Latino-Am Enferm 2006; 14(pt.2):199-206.

26. Santos SR, Schor N. Vivências da maternidade na adolescência precoce. Cad Saude Publica 2003; 37(pt. 1):15-23.

27. Cabral CS. Contracepção e gravidez na adolescência na perspectiva de jovens pais de uma comunidade favelada do Rio de Janeiro. Cad Saude Publica 2003; 19(Supl. 2):283-292.

28. Jardim DP, Bretas JRS. Orientação sexual na escola: a concepção dos professores de Jandira - SP. Rev Bras Enferm 2006; 59(2):157-162.

29. Berlofi LM, Alkmin ELC, Berbiere M, Guazzelli CA, Araújo FF. Prevenção de reincidência de gravidez em adolescentes: Efeitos de um programa de Planejamento Familiar. Acta Paul Enferm 2006 19(pt. 2):196-200. 
30. Alves CA, Brandão ER. Vulnerabilidades no uso de métodos contraceptivos entre adolescentes e jovens: interseções entre políticas públicas e atenção à saúde. Cien Saude Colet 2009; 14(2):661-670.

31. Vieira LM, Saes SO, Dória APB, Goldberg TBL. Reflexões sobre a anticoncepção na adolescência no Brasil. Rev Bras Saúde Matern Infant 2006; 6(pt. 1):135-140.

32. Taquette SR, Vilhena MM, Silvia MM, Vale MP. Conflitos éticos no atendimento à saúde de adolescentes. Cad Saude Publica 2005; 21(pt. 6):1717-1725.

33. Costa MCO, Formigli VLA. Avaliação da qualidade de serviço de saúde para adolescentes. Rev Saude Publica 2001; 35(2):177-184.

34. Villela W, Doreto DT. Sobre a experiência sexual dos jovens. Cad Saude Publica 2006; 22(pt.11):9.

35. Almeida AFF, Hardy E .Vulnerabilidade de gênero para a paternidade em homens adolescentes. Rev Saude Publica 2007; 41(pt. 4):565-572.

36. Taquette SR, organizadora. Aids e juventude: gênero, classe e raça. Rio de Janeiro: EdUERJ; 2009.

37. Taquette SR, Vilhena MM, Paula MC. Doenças sexualmente transmissíveis e gênero: um estudo transversal com adolescentes no Rio de Janeiro. Cad Saude Publica 2004; 20(1):282-290.

38. Brêtas JRS, Ohara CVS, Jardim DP. O comportamento sexual de adolescentes em algumas escolas no município de Embu, São Paulo, Brasil. Rev Gaúcha Enferm 2008; 29(4):581-587.

39. Paiva V, Calazans G, Venturi G, Dias R. Idade e uso de preservativo na iniciação sexual de adolescentes brasileiros. Rev Saude Publica 2008; 42(Supl. 1):45-53.

40. Olinto MTA, Galvão LW. Características reprodutivas de mulheres de 15 a 49 anos: estudos comparativos e planejamento de ações. Rev Saude Publica 1999; 33(1):64-72.

41. Rozenberg R. Práticas contraceptivas de adolescentes brasileiras: análise da Pequisa Nacional de Demografia e Saúde - 2006 [dissertação]. Rio de Janeiro: Instituto Fernandes Figueira, Fundação Oswaldo Cruz; 2010.

42. Leite IC, Rodrigues RN, Fonseca MC. Fatores associados com o comportamento sexual e reprodutivo entre adolescentes das regiões Sudeste e Nordeste do Brasil. Cad Saude Publica 2004; 20(2):474-481.

43. Vieira LM, Goldberg TBL, Saes SO, Doria AAB. Abortamento na adolescência: um estudo epidemiológico. Cien Saude Colet 2007; 12(5):1201-1208.

44. Guimarães AMAN, Vieira MJ, Palmeira JA. Informações dos adolescentes sobre métodos anticoncepcionais. Rev Latino-am Enfermagem 2003; 11(3): 293-298.

45. Brasil. Ministério da Saúde (MS). Secretaria de Vigilância Saúde. Programa Nacional de DST e Aids. Pesquisa de Conhecimento Atitudes e Práticas na População Brasileira de 15 a 54 anos, 2004. Brasília: MS; 2005.

Artigo apresentado em 05/09/2011

Aprovado em 05/11/2011

Versão final apresentada em 30/11/2011 
